\title{
Crystal Structures and Coordination Behavior of Aqua- and Cyano-Co(III) Tetradehydrocorrins in the Heme Pocket of Myoglobin
}

Yoshitsugu Morita, ${ }^{\dagger}$ Koji Oohora, ${ }^{\dagger}{ }^{\dagger}$ Eiichi Mizohata, ${ }^{\dagger}$ Akiyoshi Sawada, ${ }^{\S}$ Takashi Kamachi, ${ }^{\S}$ Kazunari Yoshizawa, ${ }^{\S}, \perp$ Tsuyoshi Inoue, ${ }^{\dagger}$ and Takashi Hayashi ${ }^{*} \dagger$

'Department of Applied Chemistry, Graduate School of Engineering, Osaka University, Suita 565-0871, Japan

${ }^{*}$ Frontier Research Base for Global Young Researchers, Graduate School of Engineering, Osaka University, Suita 565-0871, Japan

${ }^{\S}$ Institute for Materials Chemistry and Engineering and International Research Center for Molecular Systems, Kyushu University, Nishi-ku, Fukuoka 819-0395, Japan

${ }^{\perp}$ Elements Strategy Initiative for Catalysts and Batteries (ESICB), Kyoto University, Nishikyo-ku, Kyoto 615-8520, Japan

\section{Contents}

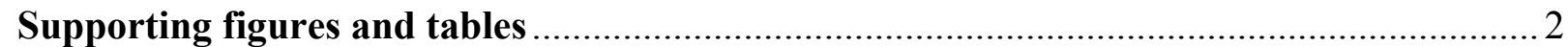

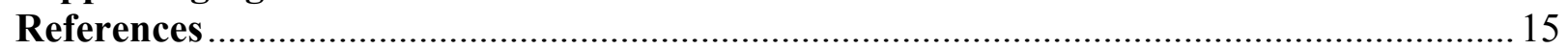




\section{Supporting figures}

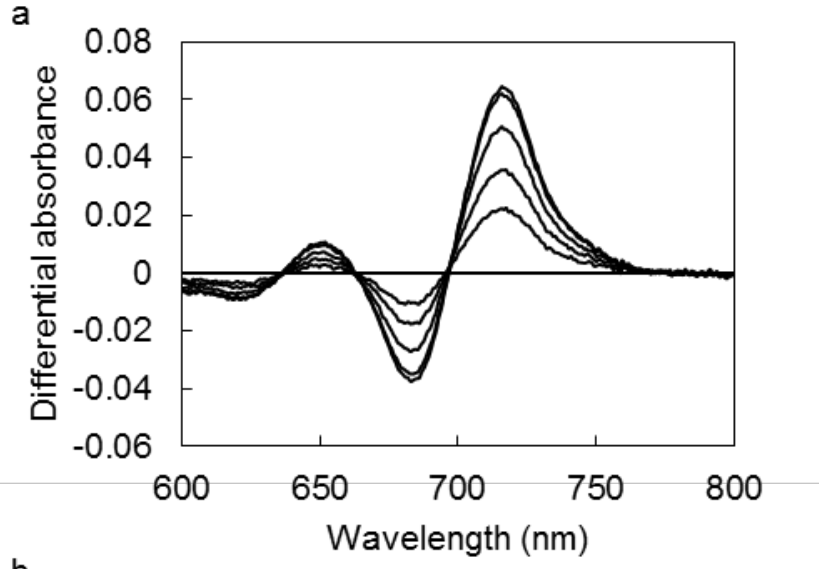

b

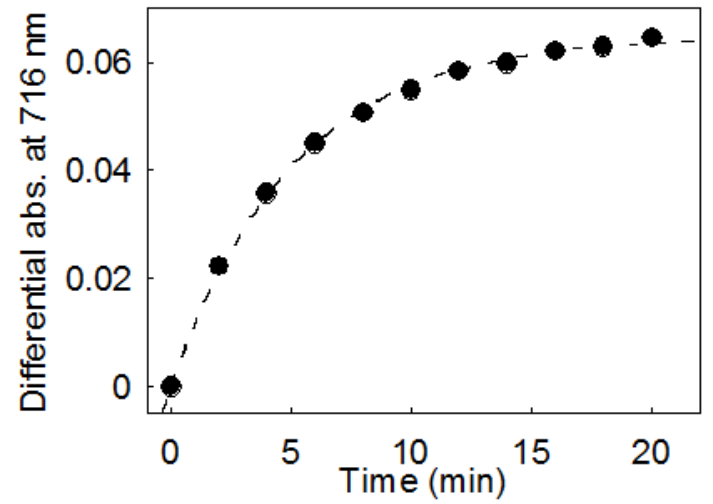

$\mathrm{C}$

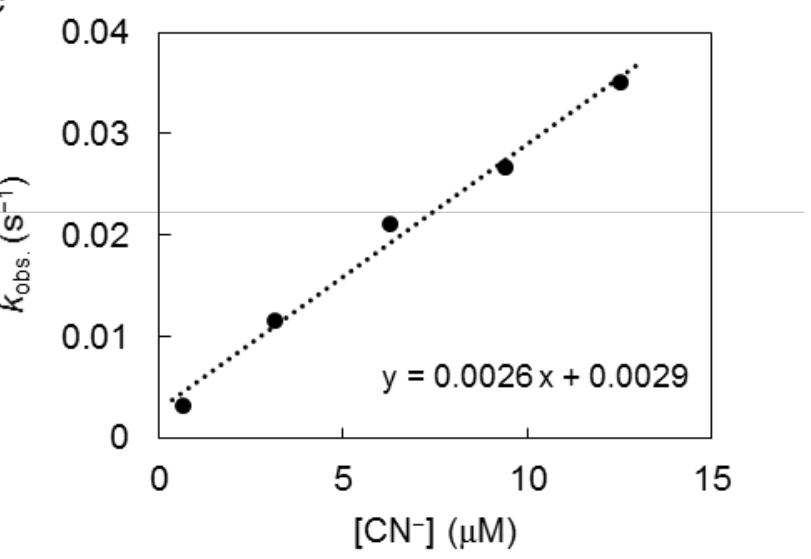

Figure S1. (a) UV-vis spectral changes of $\mathrm{rMb}\left(\mathrm{Co}^{\mathrm{III}}\left(\mathrm{OH}_{2}\right)(\mathrm{TDHC})\right)(10 \mu \mathrm{M})$ in $0.1 \mathrm{M}$ potassium phosphate buffer at $\mathrm{pH} 7.0$ at $25^{\circ} \mathrm{C}$ upon addition of $\mathrm{KCN}(0.1 \mathrm{mM})$ for $0,2,4,8,16$ and $20 \mathrm{~min}$. (b) Time course of absorption changes at $716 \mathrm{~nm}$, which is $\lambda_{\max }$ of $\mathrm{rMb}\left(\mathrm{Co}{ }^{\mathrm{III}}(\mathrm{CN})(\mathrm{TDHC})\right)$, in Figure S1a. From the fitting curve, the rate constant of $k_{\text {obs. }}$ is determined to be $0.20 \mathrm{~min}^{-1}$. (c) Relationship of the relaxation rate with the cyanide concentration. The values of $k_{\text {on }}=0.0026$ $\mu \mathrm{M}^{-1} \mathrm{~s}^{-1}$ and $k_{\text {off }}=0.0029 \mathrm{~s}^{-1}$ were obtained from the plots analyzed with the following equation. ${ }^{\mathrm{S} 1} k_{\text {obs. }}=k_{\text {off }}+k_{\text {on }}[\mathrm{L}]$. The binding constant of cyanide for $\mathrm{rMb}\left(\mathrm{Co}^{\mathrm{III}}\left(\mathrm{H}_{2} \mathrm{O}\right)(\mathrm{TDHC})\right)$ was determined to be $K=k_{\text {on }} / k_{\text {off }}=8.9 \times 10^{5} \mathrm{M}^{-1}$, which is significantly lower than that of cyanocobalamin $(\mathrm{CNCbl})\left(K \geq 10^{12} \mathrm{M}^{-1}\right){ }^{\mathrm{S} 2}$ 


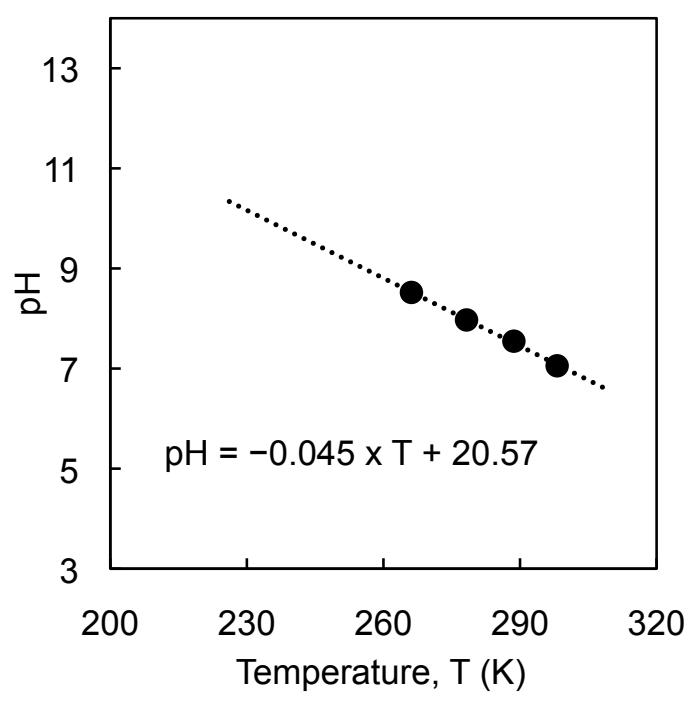

Figure S2. Plots of the $\mathrm{pH}$ value of the reservoir solution against the temperature. 
a
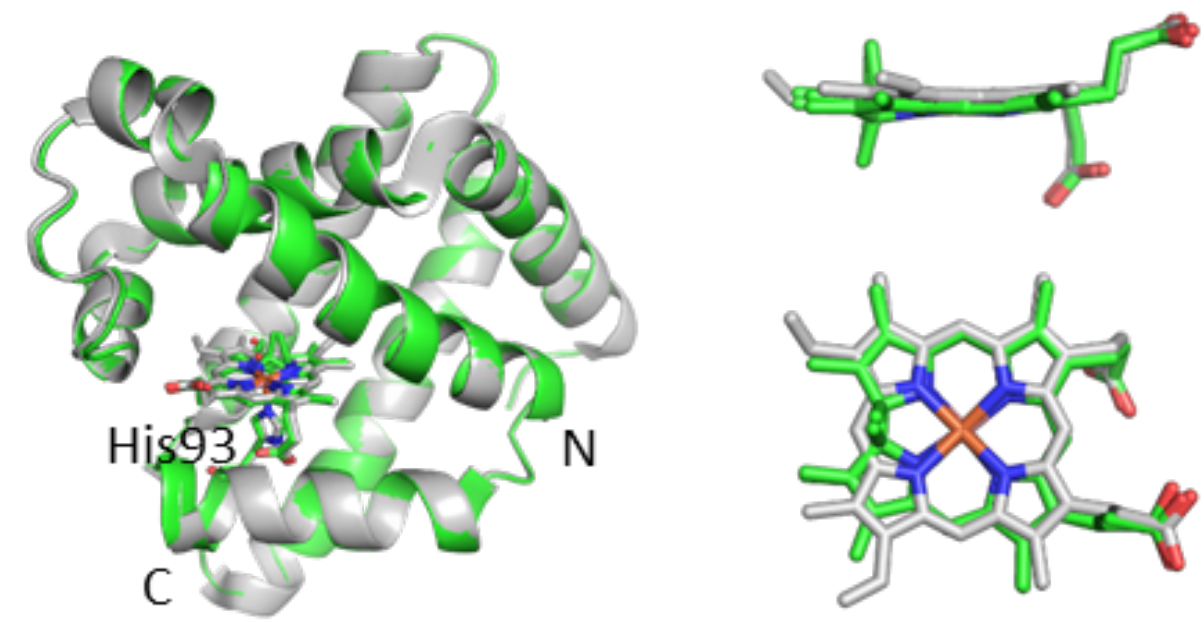

b
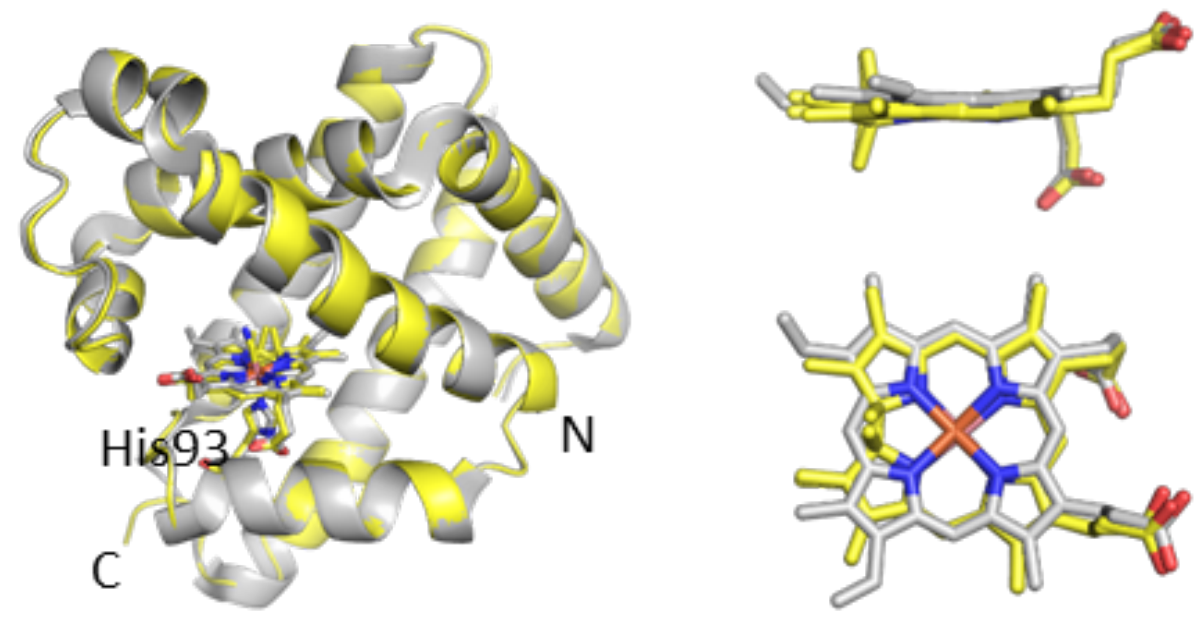

Figure S3. Superimpositions of native myoglobin (PDB ID: 2V1K) (gray) ${ }^{\mathrm{S3}}$ and the myoglobins reconstituted with (a) $\mathrm{Co}^{\mathrm{III}}(\mathrm{HO})(\mathrm{TDHC})$ (green) and (b) $\mathrm{Co}^{\mathrm{III}}(\mathrm{CN})(\mathrm{TDHC})$ (yellow). The whole structures (left), and side view and top view of the cofactors (right) in the reconstituted myoglobins are shown. 
a

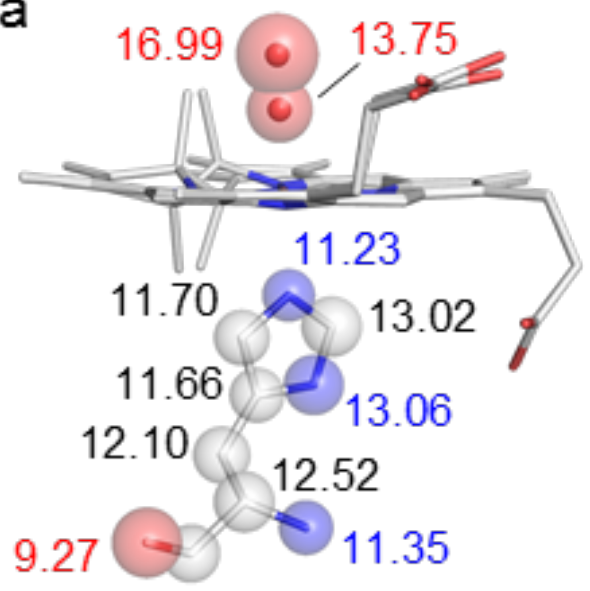

11.77

C

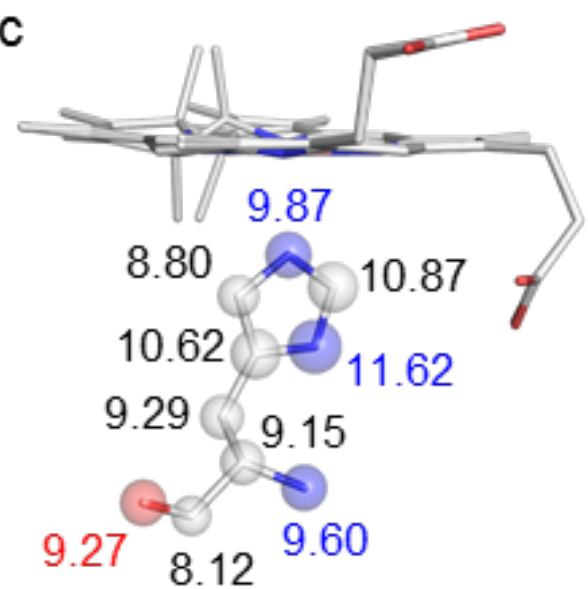

b

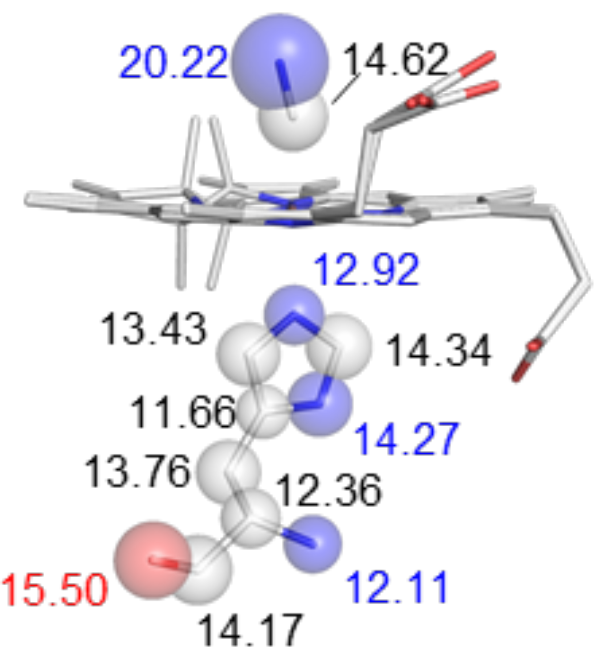

d

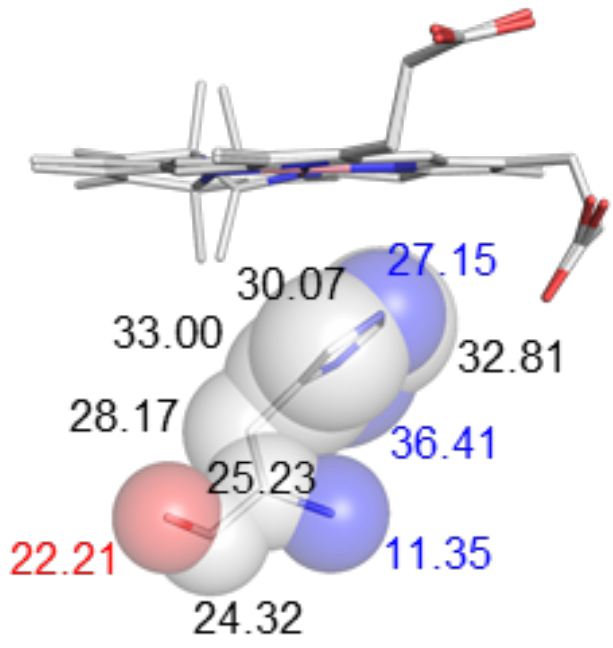

Figure S4. Variations in crystallographic $B$-factors of the axial ligands of the reconstituted proteins are represented as both the value and sphere size of each atom: (a) $\mathrm{rMb}\left(\mathrm{Co}^{\mathrm{III}}(\mathrm{OH})(\mathrm{TDHC})\right)$, (b) $\mathrm{rMb}\left(\mathrm{Co}{ }^{\mathrm{III}}(\mathrm{CN})(\mathrm{TDHC})\right),(\mathrm{c}) \mathrm{rMb}\left(\mathrm{Co}^{\mathrm{II}}(\mathrm{TDHC})\right)^{\mathrm{S} 4}$ and (d) $\mathrm{rMb}\left(\mathrm{Co}^{\mathrm{I}}(\mathrm{TDHC})\right)^{\mathrm{S} 4}$. 

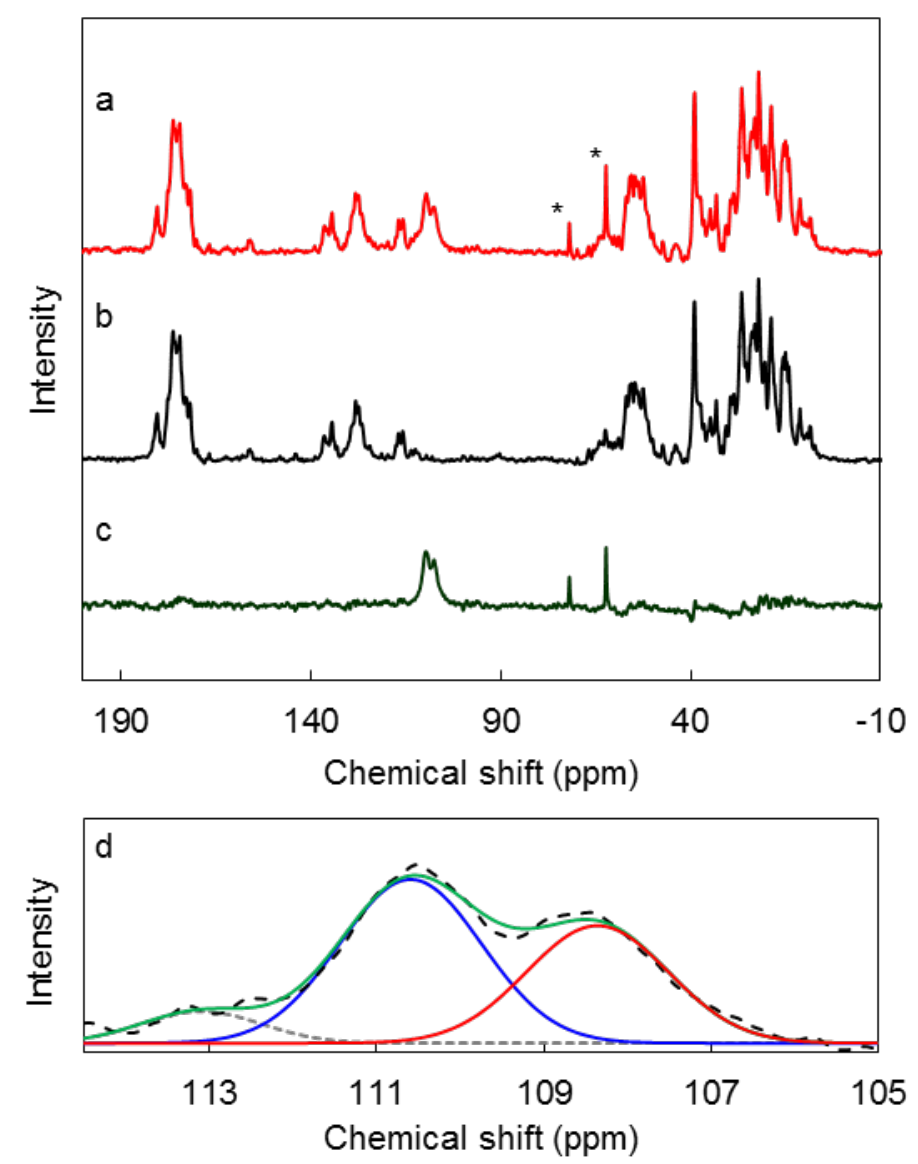

Figure S5. ${ }^{13} \mathrm{C}$ NMR $(150 \mathrm{MHz})$ spectra of $\mathrm{rMb}\left(\mathrm{Co}^{\mathrm{III}}(\mathrm{CN})(\mathrm{TDHC})\right)$ prepared with (a) ${ }^{13} \mathrm{C}$ enriched and (b) non-enriched $\mathrm{KCN}$ in $0.1 \mathrm{M}$ potassium phosphate buffer at $\mathrm{pH} 7.0$ containing $10 \% \mathrm{D}_{2} \mathrm{O}$. The asterisks identify the peaks of grecerin at $63.17 \mathrm{ppm}$ and $71.90 \mathrm{ppm}$ as a reference. The chemical shift of grecerin was reported to be $63.17 \mathrm{ppm}$ from TMS. ${ }^{\mathrm{S} 5}$ (c) The differential spectrum of $\mathrm{a}-\mathrm{b}$. (d) Expanded NMR spectrum of $\mathrm{rMb}\left(\mathrm{Co}{ }^{\mathrm{III}}\left({ }^{13} \mathrm{CN}\right)(\mathrm{TDHC})\right.$ ) (in black dash line) with simulated spectra of the peaks at $108.4 \mathrm{ppm}$ (in red solid line), $110.6 \mathrm{ppm}$ (in blue solid line) and $112.3 \mathrm{ppm}$ (gray dashed line), and the sum of the simulated spectra (in green solid line) for the determination of the ratio of the areas. 


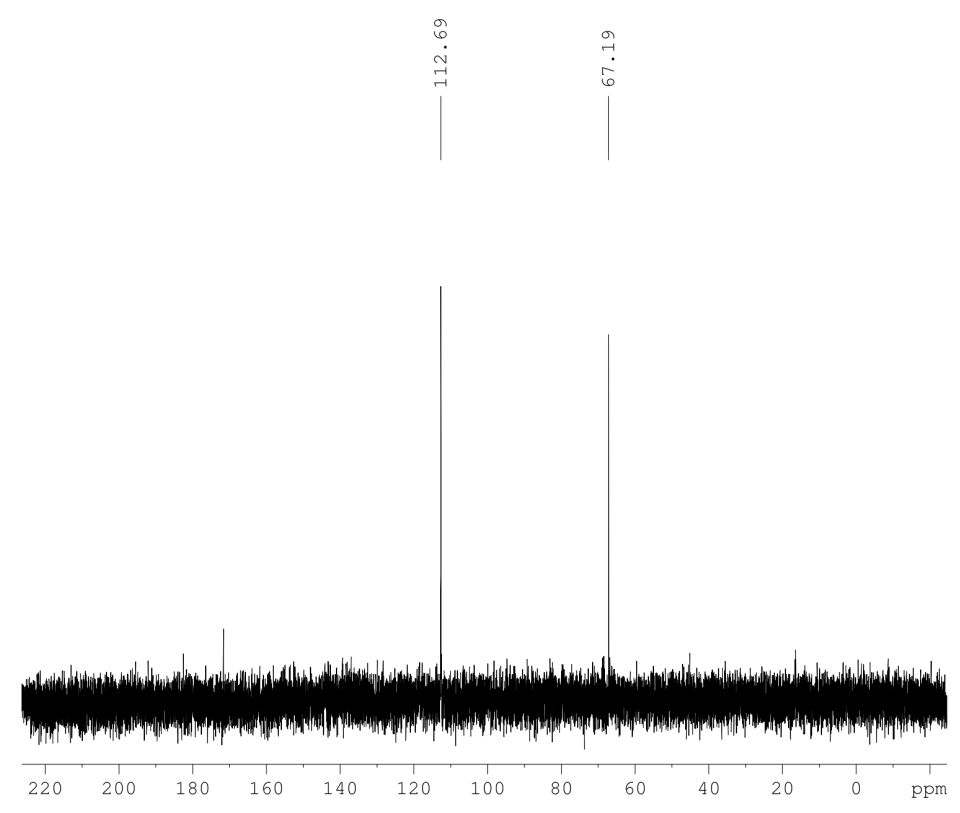

Figure S6. ${ }^{13} \mathrm{C}$ NMR $(150 \mathrm{MHz})$ spectrum of $\mathrm{Co}\left({ }^{13} \mathrm{CN}\right)_{2}(\mathrm{TDHC})$ in $0.1 \mathrm{M}$ potassium phosphate buffer containing $10 \% \mathrm{D}_{2} \mathrm{O}$ and 1,4-dioxane $(25 \mathrm{mM})$ as a reference. The chemical shift of 1,4dioxane was reported to be $67.19 \mathrm{ppm}$ from TMS. ${ }^{\mathrm{S} 5}$ The complex was prepared by autoxidation of $\mathrm{Co}^{\mathrm{II}}(\mathrm{TDHC})(2 \mathrm{mM})$ in the same buffer with $\mathrm{K}^{13} \mathrm{CN}(4 \mathrm{mM})$ under aerobic conditions. ${ }^{\mathrm{S} 6}$

a

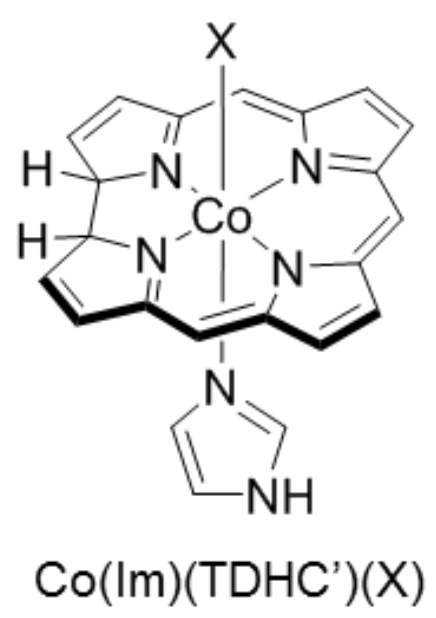

b

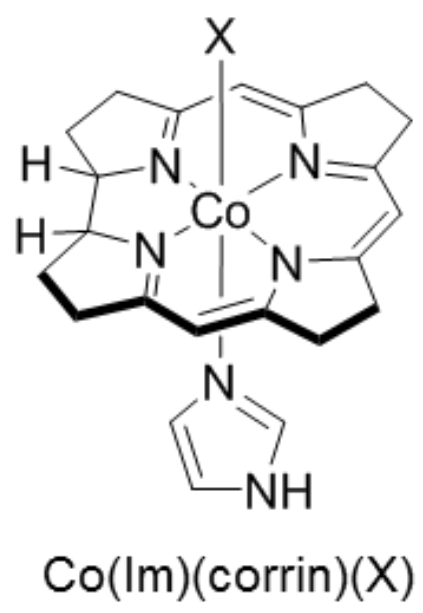

Figure S7. Chemical structures of the imidazole-coordinated (a) $\mathrm{Co}\left(\mathrm{TDHC}^{\prime}\right)(\mathrm{X})$ and (b) $\mathrm{Co}($ corrin $)(\mathrm{X})$ as models of $\mathrm{Co}(\mathrm{TDHC})$ and cobalamin, respectively. 

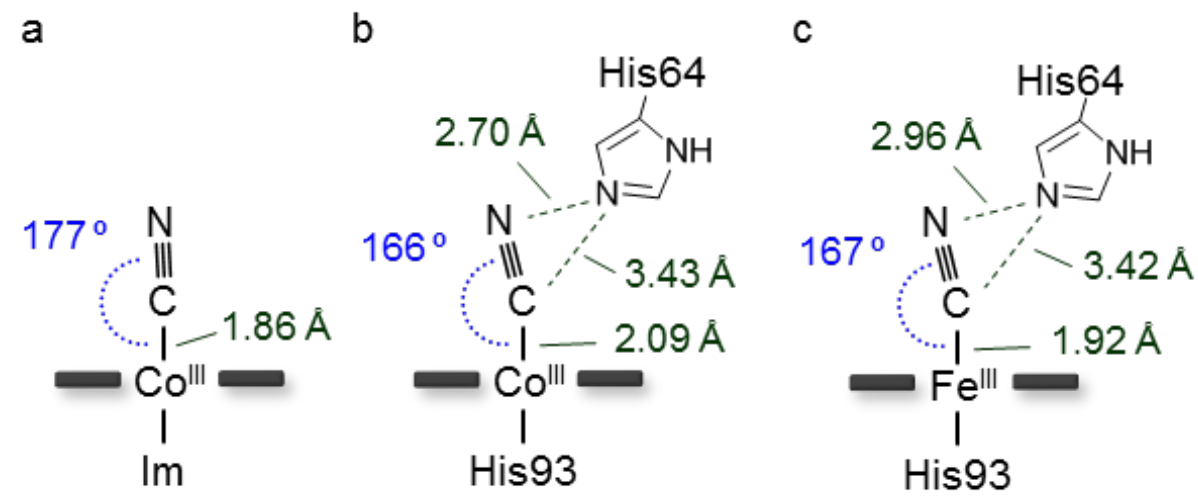

Figure S8. Schematic representations for (a) DFT-optimized structure of $\mathrm{Co}^{\mathrm{III}}(\mathrm{CN})(\mathrm{Im})(\mathrm{TDHC})$, and crystal structures of (b) $\mathrm{rMb}\left(\mathrm{Co}^{\mathrm{III}}(\mathrm{CN})(\mathrm{TDHC})\right.$ ) and (c) cyano-nMb (PDB ID: $\left.2 \mathrm{JHO}\right){ }^{\mathrm{S} 7}$ 
Table S1. DFT-optimized Bond Lengths, Atomic Charges, Stretching Frequencies and BDEs of $\mathrm{Co}^{\mathrm{III}}(\mathrm{CN})(\mathrm{Im})\left(\mathrm{TDHC}^{\prime}\right)$ and $\mathrm{Co}^{\mathrm{III}}(\mathrm{CN})(\mathrm{Im})\left(\right.$ corrin). ${ }^{a}$

\begin{tabular}{lcccccc}
\hline \multirow{2}{*}{ compound } & \multicolumn{3}{c}{ bond length $(\AA)$} & $\begin{array}{c}\text { atomic charge } \\
\text { on the carbon } \\
\text { atom of cyanide }\end{array}$ & $\begin{array}{c}\text { stretching fre- } \\
\text { quency of cyanide } \\
\left(\mathrm{cm}^{-1}\right)\end{array}$ & $\begin{array}{c}\text { BDE of } \\
\mathrm{Co}-\mathrm{N}(\mathrm{Im}) \\
(\mathrm{kcal} / \mathrm{mol})\end{array}$ \\
\hline $\mathrm{Co}^{\mathrm{III}}(\mathrm{CN})(\mathrm{Im})\left(\mathrm{TDHC}{ }^{\prime}\right)$ & 1.88 & 1.85 & 2.08 & 0.282 & 2170 & 20.5 \\
$\mathrm{Co}^{\mathrm{III}}(\mathrm{CN})(\mathrm{Im})($ corrin) & 1.91 & 1.84 & 2.05 & 0.278 & 2165 & 22.0
\end{tabular}

${ }^{a}$ at the BP86/6-31G(d) level of theory. An average distance between the Co and equatorial $\mathrm{N}$ $\left(\mathrm{N}_{\text {eq }}\right)$ atoms of $\mathrm{Co}$ (TDHC') $(1.88 \AA)$ is shorter than that of Co(corrin) $(1.91 \AA)$ because the four additional double bonds at the $\beta$-positons of all of the pyrroles of $\mathrm{Co}$ (TDHC') provides a smaller framework size than that of $\mathrm{Co}$ (corrin). This results in longer $\mathrm{Co}-$ axial ligand bonds of $\mathrm{Co}^{\text {III }}(\mathrm{CN})(\mathrm{Im})\left(\mathrm{TDHC}^{\prime}\right)$ than those of $\mathrm{Co}^{\text {III }}(\mathrm{CN})(\mathrm{Im})($ corrin).

Table S2. Cartesian coordinates for $\mathrm{Co}^{\mathrm{III}}\left(\mathrm{OH}_{2}\right)(\mathrm{Im})\left(\mathrm{TDHC}^{\prime}\right)$.

$\begin{array}{lrrr}\mathrm{C} & 0.114703 & 0.396579 & 3.741820 \\ \mathrm{~N} & 0.203762 & -0.976013 & 3.598107 \\ \mathrm{C} & 0.027372 & 0.903888 & 2.465824 \\ \mathrm{C} & 0.173785 & -1.288452 & 2.279697 \\ \mathrm{~N} & 0.065442 & -0.156677 & 1.562758 \\ \mathrm{H} & 0.288124 & -1.652828 & 4.358734 \\ \mathrm{H} & -0.050724 & 1.938559 & 2.145702 \\ \mathrm{H} & 0.235942 & -2.299870 & 1.887787 \\ \mathrm{C} & 3.438548 & -2.319722 & -0.567967 \\ \mathrm{C} & 2.496638 & -3.308297 & -0.588945 \\ \mathrm{C} & 1.185655 & -2.660260 & -0.543886 \\ \mathrm{~N} & 1.373387 & -1.277546 & -0.508872 \\ \mathrm{C} & 2.728231 & -1.042136 & -0.497997 \\ \mathrm{C} & 3.307540 & 0.233308 & -0.418214 \\ \mathrm{C} & -0.056009 & -3.296975 & -0.508812 \\ \mathrm{C} & -1.290424 & -2.578996 & -0.536143\end{array}$




$\begin{array}{lrrr}\mathrm{N} & -1.308531 & -1.242834 & -0.653895 \\ \mathrm{C} & -2.670194 & -3.081412 & -0.489544 \\ \mathrm{C} & -3.517088 & -2.018136 & -0.575484 \\ \mathrm{C} & -2.698498 & -0.774719 & -0.798379 \\ \mathrm{C} & -2.761613 & 0.526702 & 0.074324 \\ \mathrm{~N} & -1.453014 & 1.155891 & -0.144087 \\ \mathrm{C} & -3.735789 & 1.650902 & -0.161191 \\ \mathrm{C} & -3.031554 & 2.802741 & -0.337910 \\ \mathrm{C} & -1.597743 & 2.473457 & -0.316789 \\ \mathrm{C} & -0.456569 & 3.331618 & -0.417285 \\ \mathrm{C} & 0.852463 & 2.855144 & -0.371784 \\ \mathrm{C} & 2.074701 & 3.657133 & -0.415776 \\ \mathrm{~N} & 1.211577 & 1.503001 & -0.315052 \\ \mathrm{C} & 2.582214 & 1.435384 & -0.352066 \\ \mathrm{C} & 3.130962 & 2.791248 & -0.391364 \\ \mathrm{H} & 4.399498 & 0.298115 & -0.431922 \\ \mathrm{H} & -0.082837 & -4.390169 & -0.492804 \\ \mathrm{H} & -0.615791 & 4.409462 & -0.510965 \\ \mathrm{H} & -2.864728 & -0.447252 & -1.847919 \\ \mathrm{H} & -2.828746 & 0.205844 & 1.135184 \\ \mathrm{H} & 2.653059 & -4.386692 & -0.643918 \\ \mathrm{H} & -2.943196 & -4.136296 & -0.414724 \\ \mathrm{H} & -4.607885 & -2.053084 & -0.620469 \\ \mathrm{H} & -4.820957 & 1.551364 & -0.088415 \\ \mathrm{H} & -3.433666 & 3.810156 & -0.465114 \\ \mathrm{H} & 2.098403 & 4.746864 & -0.464151 \\ \mathrm{Co} & 0.016652 & 0.032295 & -0.329587 \\ \mathrm{O} & 0.158675 & 0.260919 & -2.387322 \\ \mathrm{H} & 4.524209 & -2.424746 & -0.598800 \\ \mathrm{H} & 4.195458 & 3.029408 & -0.419140 \\ \mathrm{H} & 0.123648 & 0.885447 & 4.713465 \\ \mathrm{H} & -0.373392 & -2.903498 \\ & -0.386871 & 1.155058 & -2.747087\end{array}$


Table S3. Cartesian coordinates for $\mathrm{Co}^{\mathrm{III}}(\mathrm{CN})(\mathrm{Im})(\mathrm{TDHC})$ '

$\begin{array}{lrrr}\mathrm{C} & 0.283270 & 0.315608 & 3.971422 \\ \mathrm{~N} & 0.754968 & -0.972293 & 3.777518 \\ \mathrm{C} & -0.031652 & 0.784622 & 2.714509 \\ \mathrm{C} & 0.718640 & -1.247723 & 2.443969 \\ \mathrm{~N} & 0.243418 & -0.195322 & 1.771096 \\ \mathrm{H} & 1.081575 & -1.606848 & 4.505719 \\ \mathrm{H} & -0.425327 & 1.756251 & 2.425763 \\ \mathrm{H} & 1.041996 & -2.191085 & 2.010078 \\ \mathrm{C} & 3.858085 & -1.406144 & -0.788325 \\ \mathrm{C} & 3.182578 & -2.593464 & -0.797161 \\ \mathrm{C} & 1.761882 & -2.284394 & -0.646805 \\ \mathrm{~N} & 1.618017 & -0.901834 & -0.540079 \\ \mathrm{C} & 2.867009 & -0.344037 & -0.620291 \\ \mathrm{C} & 3.122414 & 1.034284 & -0.541166 \\ \mathrm{C} & 0.716884 & -3.206434 & -0.594182 \\ \mathrm{C} & -0.652540 & -2.808441 & -0.531095 \\ \mathrm{~N} & -0.988502 & -1.519172 & -0.495922 \\ \mathrm{C} & -1.881937 & -3.619553 & -0.552389 \\ \mathrm{C} & -2.950686 & -2.777174 & -0.578916 \\ \mathrm{C} & -2.431466 & -1.368701 & -0.686807 \\ \mathrm{C} & -2.815815 & -0.170277 & 0.248571 \\ \mathrm{~N} & -1.693939 & 0.763784 & 0.074428 \\ \mathrm{C} & -4.031763 & 0.686209 & 0.018706 \\ \mathrm{C} & -3.626267 & 1.971685 & -0.160414 \\ \mathrm{C} & -2.156490 & 1.998838 & -0.139731 \\ \mathrm{C} & -1.269474 & 3.105280 & -0.295653 \\ \mathrm{C} & 0.115999 & 2.967578 & -0.304995 \\ \mathrm{C} & 1.093661 & 4.045631 & -0.434632 \\ \mathrm{C} & 0.798771 & 1.753572 & -0.233328 \\ \mathrm{H} & 2.133895 & 2.019906 & -0.394199 \\ \mathrm{H} & & 3.466966 & -0.478637 \\ \mathrm{H} & -.359808 & -0.551932 & 1.290686\end{array}$




$\begin{array}{lrrr}\mathrm{H} & 3.588361 & -3.599871 & -0.909334 \\ \mathrm{H} & -1.900917 & -4.711417 & -0.567173 \\ \mathrm{H} & -4.003707 & -3.056222 & -0.650569 \\ \mathrm{H} & -5.060291 & 0.325917 & 0.086499 \\ \mathrm{H} & -4.258312 & 2.850913 & -0.301749 \\ \mathrm{H} & 0.842027 & 5.105316 & -0.493877 \\ \mathrm{Co} & -0.011015 & 0.036958 & -0.283220 \\ \mathrm{C} & -0.220134 & 0.307525 & -2.098654 \\ \mathrm{H} & 4.932080 & -1.241600 & -0.887727 \\ \mathrm{H} & 3.299290 & 3.956154 & -0.583913 \\ \mathrm{H} & 0.221257 & 0.769477 & 4.957614 \\ \mathrm{~N} & -0.415070 & 0.463010 & -3.252656\end{array}$

Table S4. Cartesian coordinates for $\mathrm{Co}^{\mathrm{III}}\left(\mathrm{CH}_{3}\right)(\mathrm{Im})\left(\mathrm{TDHC}^{\prime}\right)$.

$\begin{array}{lrrr}\mathrm{C} & 0.137243 & 0.392941 & 3.944804 \\ \mathrm{~N} & 0.675589 & -0.875035 & 3.798618 \\ \mathrm{C} & -0.172828 & 0.806667 & 2.666441 \\ \mathrm{C} & 0.677523 & -1.186765 & 2.470137 \\ \mathrm{~N} & 0.166799 & -0.182483 & 1.754094 \\ \mathrm{H} & 1.017359 & -1.471219 & 4.551692 \\ \mathrm{H} & -0.605796 & 1.750968 & 2.342750 \\ \mathrm{H} & 1.057728 & -2.125904 & 2.073568 \\ \mathrm{C} & 3.840849 & -1.480449 & -0.692944 \\ \mathrm{C} & 3.144211 & -2.656908 & -0.698366 \\ \mathrm{C} & 1.726133 & -2.318096 & -0.635445 \\ \mathrm{~N} & 1.596736 & -0.927856 & -0.593791 \\ \mathrm{C} & 2.863350 & -0.398009 & -0.615748 \\ \mathrm{C} & 3.139962 & 0.976683 & -0.545588 \\ \mathrm{C} & 0.661766 & -3.218753 & -0.587101 \\ \mathrm{C} & -0.697967 & -2.794765 & -0.581214 \\ \mathrm{~N} & -1.010955 & -1.495738 & -0.637724 \\ \mathrm{C} & -1.936944 & -3.589287 & -0.541444 \\ \mathrm{C} & -2.996142 & -2.738418 & -0.601658 \\ \mathrm{C} & -2.463178 & -1.343003 & -0.793883 \\ \mathrm{C} & -2.801985 & -0.114886 & 0.113269 \\ \mathrm{~N} & -1.671670 & 0.794937 & -0.124238\end{array}$




$\begin{array}{lrrr}\mathrm{C} & -4.014596 & 0.756408 & -0.082014 \\ \mathrm{C} & -3.594979 & 2.036242 & -0.268834 \\ \mathrm{C} & -2.123789 & 2.044009 & -0.292101 \\ \mathrm{C} & -1.219772 & 3.135274 & -0.428547 \\ \mathrm{C} & 0.164662 & 2.966682 & -0.416090 \\ \mathrm{C} & 1.165443 & 4.026062 & -0.489273 \\ \mathrm{~N} & 0.816310 & 1.732912 & -0.356467 \\ \mathrm{C} & 2.164656 & 1.980509 & -0.445022 \\ \mathrm{C} & 2.391776 & 3.422664 & -0.496255 \\ \mathrm{H} & 4.187702 & 1.288064 & -0.596724 \\ \mathrm{H} & 0.883083 & -4.289767 & -0.590261 \\ \mathrm{H} & -1.621332 & 4.147533 & -0.527173 \\ \mathrm{H} & -2.668617 & -1.025969 & -1.839013 \\ \mathrm{H} & -2.759774 & -0.466631 & 1.165383 \\ \mathrm{H} & 3.537250 & -3.673508 & -0.746931 \\ \mathrm{H} & -1.969057 & -4.679634 & -0.483239 \\ \mathrm{H} & -4.053790 & -3.006941 & -0.637044 \\ \mathrm{H} & -5.045540 & 0.412419 & 0.023263 \\ \mathrm{H} & -4.218844 & 2.925967 & -0.379798 \\ \mathrm{H} & 0.937782 & 5.092148 & -0.530469 \\ \mathrm{Co} & -0.018916 & 0.036172 & -0.432904 \\ \mathrm{C} & -0.091614 & 0.332900 & -2.387252 \\ \mathrm{H} & -0.765326 & 1.177952 & -2.604542 \\ \mathrm{H} & 0.926482 & 0.565739 & -2.735078 \\ \mathrm{H} & -0.450565 & -0.578195 & -2.892072 \\ \mathrm{H} & 4.922089 & -1.338613 & -0.733028 \\ \mathrm{H} & 3.374132 & 3.894876 & -0.547833 \\ \mathrm{H} & 0.033429 & 0.870703 & 4.916270\end{array}$

Table S5. Cartesian coordinates for $\mathrm{Co}^{\mathrm{III}}(\mathrm{CN})(\mathrm{Im})($ corrin).

$\begin{array}{llll}\mathrm{C} & -1.065617 & 2.782519 & -0.403828 \\ \mathrm{~N} & -1.346695 & 1.426570 & -0.283523 \\ \mathrm{C} & -2.690480 & 1.220668 & -0.355700 \\ \mathrm{C} & -3.455536 & 2.528858 & -0.405740 \\ \mathrm{C} & -2.342784 & 3.575115 & -0.617312\end{array}$




\begin{tabular}{|c|c|c|c|}
\hline C & -3.307857 & -0.029633 & -0.392860 \\
\hline C & -2.652391 & -1.262581 & -0.434131 \\
\hline $\mathrm{N}$ & -1.304544 & -1.433675 & -0.393827 \\
\hline C & -0.991611 & -2.786484 & -0.446252 \\
\hline C & -2.255353 & -3.628377 & -0.483567 \\
\hline C & -3.386341 & -2.582880 & -0.558880 \\
\hline C & 0.282870 & -3.315460 & -0.451186 \\
\hline C & 1.457962 & -2.510747 & -0.475933 \\
\hline$N$ & 1.415803 & -1.196671 & -0.412597 \\
\hline C & 2.750349 & -0.601017 & -0.681784 \\
\hline C & 3.720751 & -1.736444 & -0.307530 \\
\hline C & 2.886911 & -3.003636 & -0.638778 \\
\hline C & 2.751975 & 0.723477 & 0.081694 \\
\hline$N$ & 1.381278 & 1.268366 & -0.112710 \\
\hline C & 1.387664 & 2.579899 & -0.225324 \\
\hline C & 2.800739 & 3.134617 & -0.154072 \\
\hline C & 3.669840 & 1.870888 & -0.388648 \\
\hline C & 0.193655 & 3.344179 & -0.367732 \\
\hline Co & -0.017123 & 0.012929 & -0.282371 \\
\hline C & 0.017639 & 0.151508 & -2.120276 \\
\hline$N$ & -0.094654 & -0.119434 & 1.763977 \\
\hline C & -0.006913 & -1.255501 & 2.556584 \\
\hline C & -0.102567 & -0.897791 & 3.884548 \\
\hline$N$ & -0.248459 & 0.479778 & 3.887169 \\
\hline C & -0.238959 & 0.915841 & 2.596677 \\
\hline $\mathrm{H}$ & -0.081024 & -1.481040 & 4.802081 \\
\hline $\mathrm{H}$ & -4.002603 & 2.683486 & 0.543699 \\
\hline $\mathrm{H}$ & -4.209404 & 2.521592 & -1.210720 \\
\hline $\mathrm{H}$ & -0.350798 & 1.069264 & 4.712524 \\
\hline $\mathrm{H}$ & 0.116724 & -2.243751 & 2.119118 \\
\hline $\mathrm{H}$ & -0.334673 & 1.959261 & 2.303816 \\
\hline $\mathrm{H}$ & 2.885799 & 0.512600 & 1.164216 \\
\hline $\mathrm{H}$ & 4.629903 & 1.899403 & 0.150171 \\
\hline $\mathrm{H}$ & 3.882845 & 1.758033 & -1.466913 \\
\hline $\mathrm{H}$ & 2.979936 & 3.583847 & 0.842918 \\
\hline $\mathrm{H}$ & 2.968547 & 3.929897 & -0.900011 \\
\hline $\mathrm{H}$ & -2.410010 & 4.439874 & 0.063974 \\
\hline $\mathrm{H}$ & -2.354034 & 3.975239 & -1.647893 \\
\hline & -4.141587 & -2.695276 & 0.238676 \\
\hline
\end{tabular}




$\begin{array}{lrrr}\mathrm{H} & -3.930468 & -2.619951 & -1.520277 \\ \mathrm{H} & -2.325882 & -4.259390 & 0.420756 \\ \mathrm{H} & -2.242480 & -4.311968 & -1.349539 \\ \mathrm{H} & 3.111136 & -3.868788 & 0.008406 \\ \mathrm{H} & 3.041027 & -3.332679 & -1.685432 \\ \mathrm{H} & 3.946692 & -1.701013 & 0.774383 \\ \mathrm{H} & 4.671512 & -1.688929 & -0.861008 \\ \mathrm{H} & 2.795733 & -0.378448 & -1.768366 \\ \mathrm{H} & 0.281801 & 4.427386 & -0.487180 \\ \mathrm{H} & -4.400467 & -0.045317 & -0.446015 \\ \mathrm{H} & 0.393427 & -4.402539 & -0.490959 \\ \mathrm{~N} & 0.053986 & 0.234784 & -3.297951\end{array}$

\section{References}

(S1) Neya, S.; Suzuki, M.; Hoshino, T.; Kawaguchi, A. T. Inorg. Chem. 2013, 52, 7387.

(S2) Hayward, G. C.; Hill, H. A.; Pratt, J. M.; Vanston, N. J.; Williams, R. J. J. Chem. Soc. Perkin 1 1965, 6485.

(S3) Hersleth, H. P.; Uchida, T.; Røhr, A. K.; Teschner, T.; Schünemann, V.; Kitagawa, T.; Trautwein, AX.; Görbitz, C. H.; Andersson, K. K. J. Biol. Chem. 2007, 282, 23372.

(S4) Hayashi, T.; Morita, Y.; Mizohata, E.; Oohora, K.; Ohbayashi, J.; Inoue, T.; Hisaeda, Y. Chem. Commun. 2014, 50, 12560.

(S5) Fulmer, G. R.; Miller, A. J. M.; Sherden, N. H.; Gottlieb, H. E.; Nudelman, A.; Stoltz, B. M.; Bercaw, J. E.; Goldberg, K. I. Organometallics 2010, 29, 2176.

(S6) Brown, K. L.; Hakimi, M. J. Inorg. Chem. 1984, 23, 1756.

(S7) Arcovito, A.; Benfatto, M.; Cianci, M.; Hasnain, S. S.; Nienhaus, K.; Nienhaus, G. U.; Savino, C.; Strange, R. W.; Vallone, B.; Della Longa, S. Proc. Natl. Acad. Sci. U. S. A. 2007, 104, 6211. 